\title{
INDEX Project: User support for buying QoS with regard to user's preferences
}

\author{
Jörn Altmann, Pravin Varaiya \\ Department of Electrical Engineering and Computer Science \\ University of California at Berkeley
}

ABSTRACT: In this article we are showing that the selection of QoS will become a difficult task for the user when he will be faced with different prices for different QoSs. Even if the user is only facing a best-effort service, he might be unable to determine the cost minimal selection of bandwidth. This article will show that the user needs support to get the best service regarding his personal situation. The mechanisms we use for such a personalized support tool (intelligent agent) are described in this article. In addition to this, the concept of a QoS architecture focusing on this problem will be developed in order to show how the agent fits into a QoS framework. The context where this investigation takes place is the INDEX ${ }^{1}$ project (INternet Demand EXperiment), a testbed for examining the user's demand and willingness to pay for different qualities of service.

\section{Introduction}

Since the need for high-speed networks increased in the last decade the cost for improving networks became a major factor. This is a change compared to the situation in earlier days, when network capacity was available from the oversupply of the telephone companies' voice networks. But, since applications require more and more bandwidth, ISPs had to build up their own high-speed networks and look for ways of covering the expenses [8]. But still today, the demand for higher bandwidth increases faster than the revenues of flat-rate pricing for covering the cost of upgrading the network. Indications of this situation can be found in the news every day. For example, the major ISPs increased the flat rate price for their customers recently [10]. But, this approach is only one possible solution to the problem.

The development in the Internet showed that the flat rate pricing and the one level of service quality aren't an appropriate model anymore. While the bandwidth consumption of the applications increases permanently, the cost for extending the high-speed networks cannot be covered by the revenues of flat rate pricing. Therefore, the introduction of differentiated services and pricing is the next step in the development of the Internet. Implementing this technology, people can be charged according to their usage of the network. In addition to this, both the consumers as well as the Internet Service Providers (ISPs) will gain an improved overall value and more cost efficient networks, respectively. From the consumer's viewpoint, the demand for differentiated services is obvious. Users have different requirements and needs for Internet services which are reflected by the kinds of applications they are using. The variety of applications range from just fetching electronic mail from a server to high-quality multimedia applications such as video conferencing. Since each of these applications requires a different bandwidth and level of QoS, price differentiation would help both of these user groups. On the one hand, if users require high-quality service for their application they would

1. This project is funded by the National Science Foundation, Hewlett Packard, Cisco Systems, SBC Communications, and the California Micro Program. get it without being disturbed by the best-effort traffic. On the other hand, the low capacity consuming users would not be required to pay more for their purposes.

Considering the situation from the ISPs' perspective, the differentiated service will solve the problem of financing the enlargement of their networks and overcome the permanent shortage of network capacity. Currently, the Internet service provision market has multiple independent service providers (ISPs) and there is strong competition for a market which is only barely segmented. Pricing will open the possibilities for ISPs to find new market niches. ISPs will get new kinds of revenue streams by supplying needs of certain groups of customers, since each customer will choose an appropriate pricing model according to his personal situation. The imaginable price models may range from flat rate to per transmitted byte charges.

Although the question of how QoS will be brought to the Internet is not solved yet, the introduction of differentiated traffic will be the next step. Recent press announcements from Cisco confirm this development [5][6]. The new software for their 7500 routers will be able to prioritize traffic into varying classes of service by application or customer and give preference to higher priority applications as multimedia applications.

However, the introduction of different quality of service levels (QoSL) and the introduction of pricing will make access to the Internet more complicated for the user. Although several QoS architectures exist which shield the applications from the complexity of the underlying QoS organization [3][4][9], the problem of how the user might express his preferences regarding prices for certain service qualities has not been addressed yet. For example, the choice of a performance-optimal service quality at a price much too high for the user could result in the user's dissatisfaction. Since the user's satisfaction with the Internet is determined by the most efficient use of the network for his special purposes, it will become necessary for the user to be supported in choosing the optimal services regarding his personal price preferences. Such support might come from a program running on the user's computer. The task of the program (which we will refer to as USP - User Support tool for selecting service-Price offers) is to fulfill two requirements. On the one hand, such a tool must be able to analyze monitored traffic caused by the user's applications in order to get information about the network performance and the kinds of application currently running [1][13]. On the other hand, the tool must be able to adjust the performance-optimal service quality to the user's purposes, taking into account his price preferences. The price preferences of the user are mainly determined by his financial situation, the time of day, and the importance of the task he has to perform.

The architecture of the USP is deployed in the INDEX project. The INDEX project, which stands for INternet Demand EXperiment project [12], is a field experiment for investigating people's willingness to pay for a certain service quality. The subjects of the 
INDEX project, who got a installation of a dedicated ISDN line between their homes and the Internet for free, have the possibility to choose between different options (i.e. as connection speeds) at different prices depending on the current experiment running.

In the next section, we will examine the problem of how the user preferences can be integrated into a QoS network in order to optimize the user's cost-performance relation for her special network usage. After presenting a general QoS network model and the INDEX network architecture, we will provide an extended QoS architecture which incorporates user specified preferences. The third section deals with the USP in more detail, with emphasis on the architecture of the USP and the methods on how to analyze the monitored network data. Finally, we will give an outlook on further research topics regarding the USP.

\section{User preferences and QoS networks}

Before incorporating a component for user preferences into a QoS architecture we will describe a general QoS network model which will have an impact on how to express user's preferences.

\subsection{QoS network model}

Beside the different pricing models, the user will face ISPs which have specialized in niche markets. Therefore, the difficulty for the user is deciding which of the ISPs is offering the service he needs. In addition to this, the user has to take care about minimizing the money he is going to spend for a service. An example for such a scenario is illustrated in Fig. 1.

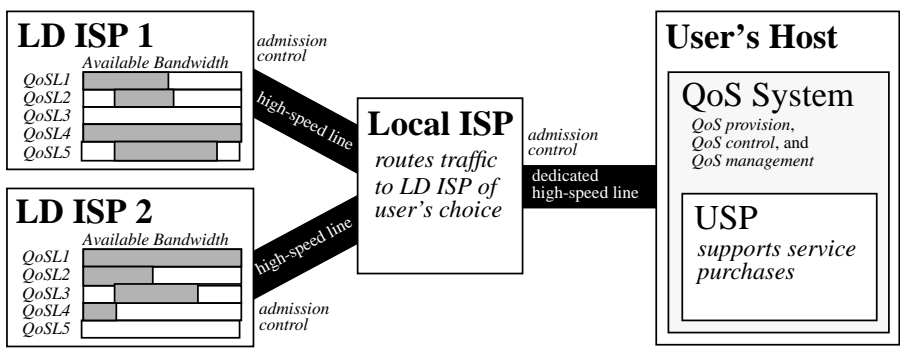

Fig. 1: General QoS network model

The example in Fig. 1 shows the user, a local ISP, and two long distance ISPs ( $L D I S P s$ ). The task of the Local ISP is to provide the user with a high-speed connection to his home. The user might pay a flat rate for this permanently installed line similar to the telephone line today. Recent developments are showing that costs for such high-speed lines will decline further and further. For instance, Covad Communication provides residents with high-speed network connections [7] at moderate prices. The two LD ISPs of Fig. 1 which forward user's packets to their destinations might offer the same service at different prices. In this case, it is up to the user to choose one of them. The user's criteria will be the available bandwidth for the required service, the satisfaction of the user with the quality of service delivered by the LD ISP in the past, and her price preferences. Choosing a consumption-based pricing model, she is charged regarding the requested service (provided that the admission control had permitted access). This scenario demonstrates that it is quite complicated for a user to keep track of all the facts and to choose the best-fitting service for his purpose if he is not an expert.

In order to clarify some possible ambiguous meanings of words, the following definitions might help. We define $Q o S$ as a combination of the basic quality metrics for the network layer: $d e$ lay, jitter, bandwidth, and reliability. When we refer to differentiated services, we are looking at the differentiation of one or more of these metrics [8]. Although these differentiated services define the so-called quality of service levels (QoSL), they don't provide any guarantees. Therefore, a distinction has to be made between three kinds of service quality: offered QoS, delivered QoS, and required QoS. Offered service quality is the service the ISP is offering during the negotiation phase of the admission testing. Delivered QoS is defined as the service actually delivered by the ISP during the data transmission. Both the offered and the delivered QoS have to meet the requirements of the required service quality which is defined as the service required by the application to run smoothly.

\subsection{Experiment environment: INDEX}

The part of the general QoS network model we are going to consider more precisely is the relationship between the ISP and the user. In order to offer attractive quality-price combinations to the user in the future, ISPs need to understand the structure of user demand. This comprises the demand for service quality as well as the demand for simplicity of service selection. Such information is collected at the INDEX project. The data reveals information about how demand varies with user experience, and whether users form discrete market segments. In addition, information will be gathered about the correlation between user application and user demand. Generally, the objectives of the INDEX project are:

- to measure user demand for Internet access as a function of QoS, pricing structure and application; and

- to demonstrate an end-to-end system that provides access to the diverse group of users at attractive price quality combinations.

The INDEX architecture is shown in Fig. 2. Each IP packet sent by the user's computer is encapsulated in a PPP frame by the ISDN router. When these packets arrive via the PSTN at one of the routers (Cisco 7507 and Cisco7513) they are tunneled to the Billing GateWay $(B G W)$. The BGW removes the PPP frame and analyses the IP packet for billing purposes. Afterwards, the IP packet is forwarded to the destination (e.g. to the Cisco 7200 router, the gateway to the Internet).

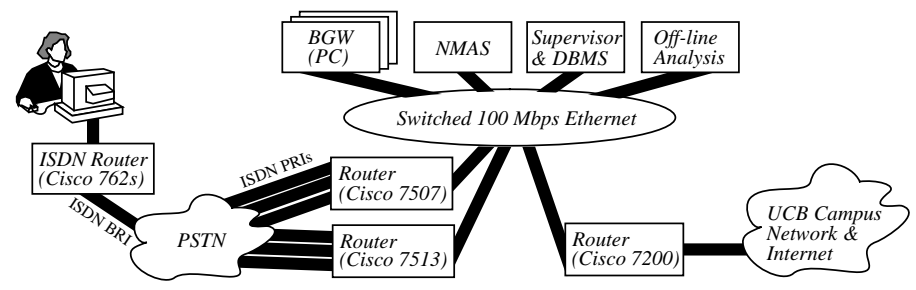

Fig. 2: The INDEX architecture

A session at the user's computer starts with the identification of the user. Once this is done, the user can select network services from a menu of service-price offerings according to his needs and demands. The behavior of the user (i.e. her choices, the initiated communications, her complaints) is monitored by different tools. The Network Measurement and Analysis System (NMAS) stores all IP packets of the last 5 minutes. In case the user complaints about bad service, the data will be written to a file for Off-line Analysis. The Supervisor process gathers data about the user's QoS-price choices, while the BGW monitors each flow. Beside the 
cumulated data about each flow, the BGW also stores data about each flow on a per minute basis.

The data collected within the INDEX project comprise detailed characteristic of each flow, as the number of packets and bytes transmitted, source and destination addresses of flows, duration of flows, user requested QoS, and type of connection such as telnet, $e$-mail, or ftp. Besides, traceroute is executed every time the user establishes a flow to a new IP address. Therefore, we can determine the number of hops to the destination host, the number of AS involved, the number of hops per AS, and whether and where unusual behavior occurred as failures and loops in routes. All these data are used for analyzing the network situation.

\subsection{USP incorporated QoS architecture}

In spite of the fact that the so called cost of service (i.e. the price the user is willing to pay for a certain QoS level) is an important aspect of QoS specification, the QoS architectures proposed in the literature [4] don't pay attention to it in an appropriate way. Since the user is concerned about how much money he is going to spend on a service, this item has to be taken into account when designing a QoS architecture.

After we demonstrated the need for a tool to support the user in his decisions with regard to his preferences, the question how such a tool fits into a general QoS architecture has to be solved. For this purpose, we took the framework described by Aurrecoechea, Campbell, and Hauw in [4] and refined the architecture for integrating the USP. Since the USP analyzes the QoS requirements of the application and the user (i.e. the QoS specification), it is part of the mapping component of the QoS architecture (see Fig. 3). In addition to this, the USP has to perform three tasks: monitoring the user's preferences, evaluating received services, and negotiating prices for a service with different ISPs. User preferences are determined by the price the user is willing to incur for a level of service depending on, for instance, the time of day, the task he has to complete, and his financial situation. The evaluation of received services helps to assess ISPs and will influence the choice of a service next time. The negotiation of prices mainly comprises the request for prices of services. But, it is imaginable to have more complicated methods of negotiating prices as auctions [2].

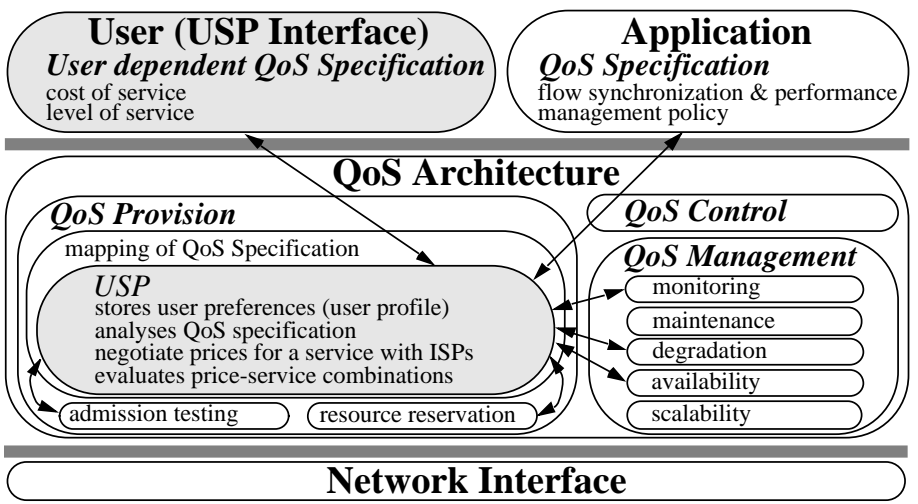

Fig. 3: USP integrated into a QoS architecture on the End-System

Fig. 3 also shows the interaction of the USP with the rest of the QoS components. As already indicated in the previous paragraph, the USP gets the QoS requirements directly from the applications and has a user interface which allows the user to give feedback to the USP on its decisions. But, in order to get information about the current situation the USP communicates with several components of the QoS management. The QoS degradation component issues a QoS indication to the USP if the QoS cannot be maintained anymore. That is the worst case. In order to even recognize slight variations of QoS, the difference between the required QoS and the delivered QoS is measured by the QoS monitoring component. These mechanisms are helpful to evaluate the price-service combination delivered by the ISP. Beside that, the USP has to check by communicating with the admission testing component and the resource reservation component whether the system and the network can provide the required QoS.

\section{Intelligent user agent as USP}

In order to achieve user support, the USP must be able to act exactly according to the user's needs and preferences. An appropriate tool is an intelligent agent which models the user's behavior. The user will benefit from such an intelligent agent since he will be relieved from the task of monitoring how much money he is spending for each flow he has initiated. The intelligent agent will negotiate prices with the agents of the Internet service providers and it will maximize the user's satisfaction by customizing the service quality to the user's price preferences.

\subsection{Structure of the USP agent}

The intelligent user agents consists of six parts (see Fig. 4). One of these is the User Interface which allows the user to interact with the agent. Two of the four panels of the interface reflect the user's usage and billing status. The values are calculated by the Billing Information Module and the Usage Information Module, respectively. The third panel represents the user's price preferences which can be changed any time. The user can give feedback to the QoS-price choices made by the agent, which will have direct impact on the selection of the QoS-price combination and will be taken into account by the agent for future decisions. This information is processed by the User Preference Module. Beside that, the user has the occasion to complain about poor service according to his subjective opinion on the fourth panel of the User Interface. The User Complaint Module is responsible for processing this data. The data entered via the User Interface is postprocessed by the Analysis Module, the central module of the agent, in order to compare received service quality with the required QoS and to evaluate services. For this purpose the Analysis Module also inquire data from a Database about previous network situations and about the user's preferences via the database interface. The Analysis Module receives data from the Communication Module and the four QoS interface modules as well. The Agent Communication Module is an interface to the agents of network providers in order to get information about QoS-price combinations and to select the best QoS-price combination for the required QoS. The four interface modules, QoS Admission/Availability Interface, QoS Degradation Interface, QoS Monitoring Interface, and QoS Specification Interface, connect to the corresponding components of the QoS architecture (see Fig. 3).

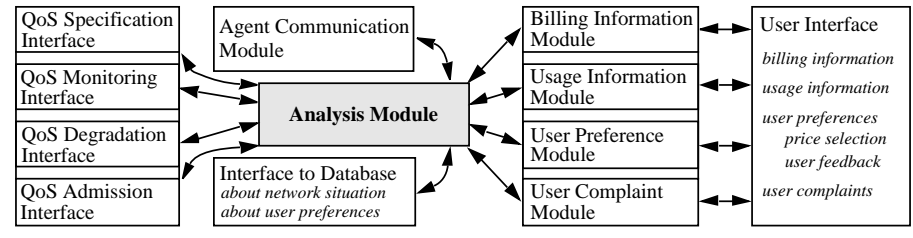

Fig. 4: Structure of the intelligent user agent 


\subsection{USP analysis Module}

The Analysis Module is the main module of the intelligent user agent. This module has to process three kinds of data in a certain order which is depicted in Fig. 5. The first type of data is generated by the QoS Specification Interface module, which in turn gets the information from the corresponding QoS component. The second type of data describes the current network situation. The Analysis Module obtains this data from the Database, the $Q o S$ Admission/Availability Interface, the QoS Degradation Interface, and the QoS Monitoring Interface. The latter three modules establish a connection with the corresponding QoS components mentioned in the QoS architecture. The third data type reflects the user's preferences. This information is either already stored in the database or is generated by the user when interacting with the User Interface.

The predictability of the agent's decisions is an important requirement, i.e. the agent's decision must be comprehensible by the user any time. The decision-making process of the agent proceeds as follows (see Fig. 5). First, the applications' QoS requirements as well as the QoS management policies of the applications are analyzed and mapped into the network layer QoS parameters. The result is a range of bandwidth of a certain QoSL for each application (see light gray area in a. of Fig. 5). These QoS requirements are compared with the ranges of QoSL bandwidths offered by different ISPs. Furthermore, the QoS requirements are compared with the QoS network situation, i.e. how was the service of this ISP in the past and how was the performance of the entire network path in the past (see gray area in b. of Fig. 5). For example, given the situation that we want to browse a WWW-page at a certain site and we already know that the connection was slow (8kbps) the last three times, then it won't be sensible to buy a bandwidth bigger than $8 \mathrm{kbps}$ this time. The results of the comparison are the bandwidth ranges marked as dark gray area in c) of Fig. 5. Finally, these bandwidth ranges are compared with the QoSL bandwidth the user is willing to pay for according to his preferences (marked as black rectangles in c. of Fig. 5).

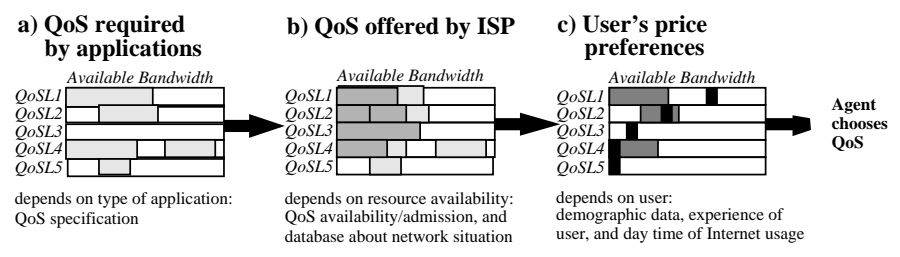

Fig. 5: Decision-making process of USP agent

In detail, the method for modeling the user's price preferences is based on statistical models and decision-making methods of the $\mathrm{AI}$ (e.g. learning decision trees). The modeling process starts with modeling the user's experience about computer networks. The result of this modeling step, and demographic data (e.g. financial situation, profession, computer experience) about the user (which helps to classify the user into one of the user groups for which the demands of Internet service is quite identical) are taken into account for processing the bandwidth preferred by the user. In addition to this input parameters, the user has the opportunity to give feedback to the agent's choice of QoS bandwidth. The feedback has direct impact on the current QoS choice as well as on future decisions of the agent. As a result of processing all these attributes, the learning decision methods suggest the QoSL bandwidth which is bought by the agent on behalf of the user.

\section{Conclusions}

Since the problem of how to deal with real money in a QoS system has not been addressed in literature yet, we showed that the user will face an too complicated situation when he is forced to manage different prices for different services. As a solution we proposed a User Support tool for selecting service-Price offers $(U S P)$, and explained how it is incorporated into a general QoS architecture. The mechanisms of the USP are based on the concept of intelligent agents. In addition to this, we described the detailed structure of the intelligent user agent as well as the mechanisms used by the main module of the agent to support the user.

Our future work will be the refinement of the methods of the intelligent user interface as well as the application of the USP in a real field experiment of the INDEX (INternet Demand EXperiment) project. In particular, we are going to investigate how the user interface has to be refined in order to increase the simplicity of such a tool. It will be particularly interesting to see, which user group will use such a tool more than others.

\section{References}

[1] Jörn Altmann, and Beat Liver, "Social Carrier Recommendation for Selecting Services in Electronic Telecommunication Markets," Policy a. Strategy for Convert. Info. Industry, 1997.

[2] Marius Busuioc, "The Requirements for Personal Mobile Assistants in a Mobile Telecommunication Environment," ECAI'96 Workshop on Intelligent Agents for Telecommunication Applications, Budapest, 1996.

[3] Roland Bless, Matthias Jacob, and Claudia Schmidt, "Service-Tailored QoS Management in High Performance Networks," 5th IEEE/IFIP IWQoS, 1997.

[4] Cristina Aurrecoechea, Andrew T. Campbell, and Linda Hauw, "A Survey of QoS Architectures," 4th IEEE/IFIP International Workshop on Quality of Service, 1996.

[5] Cisco Systems Inc., "Cisco Systems and Belle Systems Develop Billing System," press release, 9th January 1998.

[6] Cisco Systems Inc., "Cisco Enables Premium Services for Internet Service Providers," press release, 3rd December 1997.

[7] Covad communications, "Covad Communications Extends Reach of its DSL Service Through Launch of ISP Partner Program," http://www.covad.com/, February 1998.

[8] Paul Ferguson, and Geoff Huston, "Quality of Service - Delivering QoS on the Internet and in Corporate Networks," Wiley Computer Publishing, January 1998.

[9] Klara Nahrstedt, and Jonathan M. Smith, "The QoS Broker,' IEEE Multimedia, vol. 2, no. 1, pp.53-67, 1995.

[10] Reuters, "Higher Toll for Net Access," San Francisco Chronicle, 1st April 1998.

[11] Scott Shenker, David Clark, Deborah Estrin, and Shai Herzog, "Pricing in Computer Networks: Reshaping the Research Agenda," Telecomm. Policy Research Conf., 1995.

[12] Pravin Varaiya, Richard Edell, and Harish Chand, "INDEX: the Internet Demand Experiment," Report of the Department of EECS, University of California, Berkeley, 1997.

[13] Bernd E. Wolfinger, "Status and Trends in Modeling Communication Systems and Computer Networks: a Survey," 2nd Polish Teletraffic Seminar, Gdansk, 1995. 